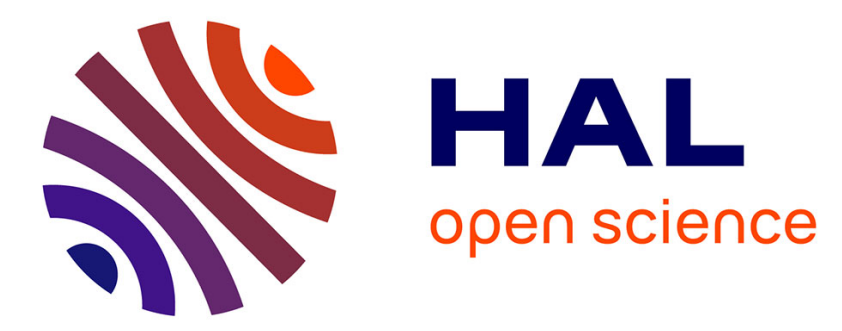

\title{
Nonprehensile Manipulation of Deformable Objects: Achievements and Perspectives from the RobDyMan Project
}

Fabio Ruggiero, Antoine Petit, Diana Serra, Aykut C Satici, Jonathan Cacace, Alejandro Donaire, Fanny Ficuciello, Luca R Buonocore, Giuseppe Andrea Fontanelli, Vincenzo Lippiello, et al.

\section{To cite this version:}

Fabio Ruggiero, Antoine Petit, Diana Serra, Aykut C Satici, Jonathan Cacace, et al.. Nonprehensile Manipulation of Deformable Objects: Achievements and Perspectives from the RobDyMan Project. IEEE Robotics and Automation Magazine, 2018, 25 (3), pp.83 - 92. 10.1109/mra.2017.2781306 . hal-01889779

\section{HAL Id: hal-01889779 \\ https://inria.hal.science/hal-01889779}

Submitted on 8 Oct 2018

HAL is a multi-disciplinary open access archive for the deposit and dissemination of scientific research documents, whether they are published or not. The documents may come from teaching and research institutions in France or abroad, or from public or private research centers.
L'archive ouverte pluridisciplinaire HAL, est destinée au dépôt et à la diffusion de documents scientifiques de niveau recherche, publiés ou non, émanant des établissements d'enseignement et de recherche français ou étrangers, des laboratoires publics ou privés. 
See discussions, stats, and author profiles for this publication at: https://www.researchgate.net/publication/323899088

\section{Nonprehensile manipulation of deformable objects: Achievements and perspectives from the RoDyMan project}

Article in IEEE Robotics \& Automation Magazine $\cdot$ March 2018

DOI: 10.1109/MRA.2017.2781306

CITATIONS

0

12 authors, including:

Fabio Ruggiero

University of Naples Federico II

48 PUBLICATIONS 510 CITATIONS

SEE PROFILE

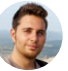

Jonathan Cacace

University of Naples Federico II

18 PUBLICATIONS 136 CITATIONS

SEE PROFILE

Some of the authors of this publication are also working on these related projects:

SHERPA: SHERPA: Smart collaboration between Humans and ground-aERial robots for imProving rescuing activities in Alpine environments. View project

Robotics: dynamics and control View project
READS

213

Aykut Satici

Boise State University

32 PUBLICATIONS 219 CITATIONS

SEE PROFILE 


\title{
Nonprehensile manipulation of deformable objects: Achievements and perspectives from the RoDyMan project
}

\author{
Fabio Ruggiero, Antoine Petit, Diana Serra, Aykut C. Satici, Jonathan Cacace, Alejandro Donaire, Fanny \\ Ficuciello, Luca R. Buonocore, G. Andrea Fontanelli, Vincenzo Lippiello, Luigi Villani, Bruno Siciliano
}

\begin{abstract}
The goal of this work is to disseminate the results achieved so far within the RODYMAN project related to planning and control strategies for robotic nonprehensile manipulation. The project aims at advancing the state of the art of nonprehensile dynamic manipulation of rigid and deformable objects to future enhance the possibility of employing robots in anthropic environments. The final demonstrator of the RODYMAN project will be an autonomous pizza maker. This article is a milestone to highlight the lessons learned so far and pave the way towards future research directions and critical discussions.
\end{abstract}

\section{OVERVIEW ABOUT NONPREHENSILE MANIPULATION IN ROBOTICS}

$\mathbf{M}$ ANIPULATING an object roughly entails changing its currentstate to a desired one. Suppose to have an object on a table that has to be moved from configuration $\mathcal{A}$ to configuration $\mathcal{B}$ on the same table. How would you move it? Maybe, the two most rated answers would be: (i) grab the object, lift it up and place it back on the table in $\mathcal{B}$; (ii) push the object on the table from configuration $\mathcal{A}$ to $\mathcal{B}$. In case (i), the object is completely grabbed between the fingertips and/or the palm, and the hand is theoretically able to resist any external disturbance wrench applied to the object. Hence form closure occurs even when infinitesimal motions of the object are prevented by the hand, otherwise force closure occurs [1]. Bilateral constraints are exhibited by the grasp in both closures. In case (ii), the object is instead pushed by one or more fingertips and these last are able to resist only to forces counteracting the direction of the pushing. Hence, nonprehensile manipulation, or graspless manipulation, occurs since only unilateral constraints are involved. These tasks can also be endorsed as dynamic when the dynamics of both the object and the robot are essential to successful task execution.

Therefore, a nonprehensile dynamic manipulation task can be generally described as a task in which the object is subject only to unilateral constraints and the dynamics of both the object and the manipulating hand, as well as the

F. Ruggiero, J. Cacace, F. Ficuciello, G.A. Fontanelli, V. Lippiello, L. Villani and B. Siciliano are with CREATE Consortium and with Department of Electrical Engineering and Information Technology, University of Naples Federico II, via Claudio 21, 80125, Naples, Italy. A. Petit is with MIMESIS team, INRIA, 1, place de l'Hopital, 67000 Strasbourg, France. D. Serra is with Rete Ferroviaria Italiana SpA, Via De'Cattani 1, 50145, Osmannoro. A.C. Satici is with Boise State University, 1910 W University Dr, Boise, United States. A. Donaire is with Queensland University of Technology, 2 George st, 4000, Brisbane, QLD, Australia. L.R. Buonocore is with CERN, Route de Meyrin 385, Geneve 23, Switzerland. related kinematics and the (quasi-)static forces play a crucial role. Pushing objects, folding clothes, carrying items on a tray, cooking in pan, performing some surgery operations are examples of nonprehensile manipulation tasks. From a robotic point of view, most of nonprehensile manipulation systems are underactuated, raising controllability challenges. However, dynamic nonprehensile manipulation benefits of several advantages such as the increase of available robot actions, bigger operative workspaces, enhanced dexterity in dynamic tasks.

On one hand, the robotics literature is well-established regarding grasping techniques [1] and control methods for manipulation tasks with grasp [2]. Within industrial applications, where simplicity and costs are relevant above all, grippers or special-purpose devices are widely used. Nevertheless, the necessity for robots working in anthropic environments is growing fast as shown by the European Strategic Resarch Agenda $^{1}$ (eSRA), where it is outlined that robots will pervade a portion of the market domain in domestic appliances, assisting living, entertainment and education. Therefore, robots should not need specific tools for each actions, but they should exploit multi-purpose devices, like multi-fingered robotic hands, and rely upon the dexterity conferred by the designed control algorithms. Manipulation dexterity is one of the main research challenge currently addressed by the robotic community. As explained above, a nonprehensile manipulation task is a dexterous task par excellence. As a matter of fact, some tasks are intrinsically prehensile like, for instance, (un)screwing a bottle cap. Other tasks can be instead tackled both in a prehensile or a nonprehensile manner, like the aforementioned example of moving an object on a table. Other tasks are inherently nonprehensile, like carrying a glass full of water on a plate. Other tasks are hybrid, in the sense that to reach the goal both prehensile and nonprehensile actions are required, like a juggler that has to repetitively catch-and-throw balls in a cascade juggling pattern.

On the other hand, the robotics literature is not fully developed for nonprehensile manipulation tasks. The classic way to cope with them is to split a task in simpler subtasks, referred to as nonprehensile manipulation primitives [3], such as throwing [4], dynamic catching [5], batting [6], juggling [7], dribbling [8], pushing [9], sliding [10], rolling [11] and so on. Each primitive, equipped with its own motion planner and controller, is then turned on and off during a complex

${ }^{1}$ https://www.eu-robotics.net/cms/upload/topic ' groups/SRA2020 'SPARC.pdf 
manipulation task by a high-level supervisor [12]. Among the mentioned nonprehensile manipulation primitives, only rolling and batting boast a considerable amount of work in the literature. There is also a lack of a general unified theoretical framework in the field, causing the continuous investigation of ad-hoc motion planners and controllers to solve the specific tasks one by one. The main reason may be found in the possible change of the contact status during a nonprehensile manipulation task, leading to non-smooth dynamics of the whole system, which complicates the control design. For this reason, dynamic nonprehensile manipulation may be considered as the most complex manipulation action, deserving attention as requested by the eSRA, and posing many research challenges to be solved.

In the described context, the RODYMAN project aims at the development of a service robot able to manipulate elastic and soft objects, as well as to manipulate both rigid and non-rigid objects in a nonprehensile way, with the ambitious goal of bridging the gap between robotic and human task execution capability. In order to reach the planned goals, three main research challenges have been identified within the RODYMAN project. Namely:

- Mechatronic development and assembly. A mobile robotic platform equipped with two commercial arms and multi-fingered hands is necessary to perform the dynamic manipulation tasks planned for the project.

- Modelling and perception. Real-time requirements posed by robot interaction with deformable objects during dynamic nonprehensile manipulation actions are essential to fulfil the required tasks.

- Control techniques for nonprehensile dynamic manipulation. The goal of the project is to advance the state of the art in controlling rigid objects in a nonprehensile way, and starting investigating the problems relative to the prehensile and nonprehensile manipulation control of deformable objects as well.

The final demonstrator of the project will be an autonomous pizza maker since preparing a pizza involves an extraordinary level of manual dexterity.

Some other projects have tried to address nonprehensile manipulation problems using different approaches. The RIBA robot $^{2}$ is able to lift up and set down patients from/to their beds and/or wheelchairs. The soft body of the robot is designed to make safe the interaction with the humans. The performed transporting task is indeed nonprehensile, but the manipulation task is not dynamic since the patient's body is considered as a rigid object, and only motion planning techniques for lifting up the body are investigated. The task is very similar to a pick-and-place operation where the transporting motion is addressed in a nonprehensile fashion. Also the ERC SHRINE project $^{3}$ goals are focused to enhance robot manipulation capabilities, so as to overcome barriers preventing robots from safe and smooth operations within anthropic environments. The sought robot dynamic manipulation, in some cases also performed in a nonprehensile way, is addressed to cooperate

\footnotetext{
${ }^{2}$ http://rtc.nagoya.riken.jp/RIBA/index-e.html
}

${ }^{3}$ http://www.shrine-project.eu

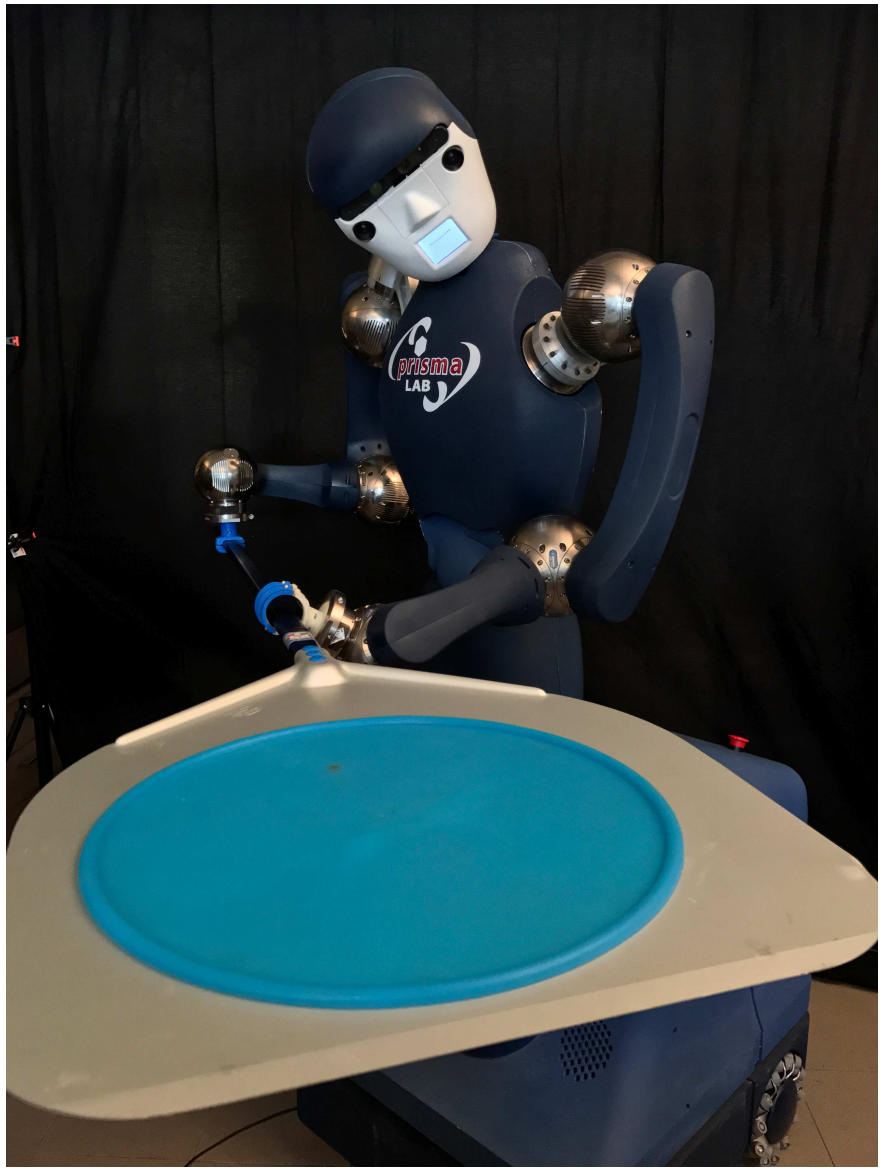

Fig. 1: The RoDYMAN platform handling the peel with two arms and two proper grippers at the end-effectors. The displayed tool is a real pizza peel employed by chefs to cook the dough in the oven. A blue silicon disk, usually employed by acrobatic pizza chefs for training, is employed in the experiments.

with humans. In the following, the results achieved so far within the RODYMAN project for the above three outlined research challenges are described. Videos of the related experiments can be found in the related YouTube channel ${ }^{4}$.

\section{ROBOT DESIGN AND ARCHITECTURE}

\section{A. Mechatronic design}

The built mechatronic set-up, referred to as RODYMAN like the project name, is a 21-degree-of-freedom (DoF) humanoidlike robot (see Fig. 1). An omnidirectional mobile platform allows the robot to move in the space. An actuated mechanism gives the ability to enlarge the support polygon during the execution of dynamic and rapid movements of the upper body. The battery pack and UPS unit used to provide power to all the devices are housed within the mobile platform, and provide at the same time the weight to stabilize the platform. Two standard PCs are also located in the base. One is a QNX-based PC used for real-time and low-level of the motors and the implementation of safety procedures. The second

\footnotetext{
${ }^{4}$ https://www.youtube.com/user/ThePRISMAlab
} 
one is a Linux-based PC used for perception and high-level planning and control algorithms. The upper-body limbs of the robot are two SCHUNK LWA 4P arms with 6 DoFs each. The seventh joint of each arm, required to add human-like redundancy, is provided by a SCHUNK PRL-100 integrated into the shoulder. To the best of the authors' knowledge, the SCHUNK were the only arms on the market to have both dimensions similar to the human arms and the control directly on the CAN bus without an external controller box. Nevertheless, experimental results show that the high friction and the low joint velocities exhibited by these arms represent a limitation on the execution of particular and complex tasks like tossing. It is worth pointing out that this solution represents only a first prototype, and the design of new arms, with advanced dynamical characteristics, is within the RODYMAN project plan.

Moreover, the dynamic model of the whole structure has been derived in a symbolic form. The LMI method in [13] has been employed to obtain the identification of the dynamic parameters by absorbing the physical constraints within the optimization procedure. Experimental results have indeed shown that friction, mostly the static part, is the dominant component in the measured torque. Therefore, a friction identification has been firstly performed separately, and then the friction parameters have been used as constraints within the LMI optimization procedure.

The RODYMAN platform is completed by two motors to actuate the torso and one for the pan-tilt neck. In order to provide enhanced dexterous manipulation skills, two anthropomorphic SCHUNK Servo-electric 5-Finger SVH hands can be applied at the end- effector tip of the two arms. However, these hands are very delicate and they are replaced with suitable 3Dprinted tools for those tasks requiring non-trivial weights in action, like the pizza-peel task described afterwards. From the perception point of view, the platform is equipped with two laser scanners in the base for odometry operation; two force sensors can be mounted on the wrist to measure the interaction forces between the end effector and the environment, while the interaction forces exerted on the robot structure can also be obtained using proper estimators [14]. Finally, the head is equipped with a stereo camera system, an RGB-D sensor and a time-of-flight camera to obtain a precise depth estimation.

\section{B. High-level software architecture}

In order to carry out the expected activities involving complex manipulation actions, a control architecture is designed to handle high-level planning tasks. A sketch of the control architecture is shown in Fig. 2, and it is described below.

The Human Robot Interaction (HRI) Interface module is used to specify high-level tasks as inputs for the system (e.g. the pizza tossing). The Supervisor module is responsible of the task decomposition process, splitting the high-level actions received by the HRI Interface in lower-level actions considering both the state of the robot and the information generated by the Perception module. After the decomposition process, each lower level action can be executed. Examples of high-level tasks are $\operatorname{Grasp}($ Object $), \operatorname{Search}($ Object $)$ or
Toss(Object), and sequences of nonprehensile manipulation primitives. In order to suitably perform the task decomposition process, the supervisor module is provided with a library of hierarchical tasks, analogous to Hierarchical Task Networks (HTNs), which can be composed by the system to achieve the desired goal [15]. In this context, if a nonprehensile manipulation action is required, the related low-level controller is invoked from the dynamic manipulation task list while the supervisor waits its termination. Otherwise, the Executors module is responsible for the action by implementing both the path and the motion planning functionalities to find an obstacle-free way for the end effectors of the robot and its base. This module relies on MoveIT! [16], a framework that integrates Universal Robot Description File, Open Motion Planning Library and other toolkits. The generated trajectories for the joints and the base of the robot are streamed to the robot actuators from the Controller modules. Finally, information about the robot environment is extracted from the Perception module via image elaboration algorithms.

The proposed high-level control architecture perfectly matches the requirements of the RODYMAN robotic platform, statically allocating the best low-level controller to accomplish desired actions. This improves the current literature since very few ways of decomposing a high-level task for nonprehensile manipulation have been developed [12]. Future directions are inclined to increase the level of collaboration of the robot with human operators allowing shared task planning and execution.

\section{PERCEPTION OF DEFORMABLE OBJECTS}

Using the point cloud data provided by the RGB-D sensor, a method to cope with real-time (35 fps) tracking of a deformable object is presented. Several contributions are proposed such as handling various large elastic deformations while ensuring physical consistency, coping with fractures, rigid motions and occlusions [17]. The case of a pizza dough being stretched and tossed is taken as example.

Since the considered system attempts to deal with large deformations and elastic volumetric strains, a realistic mechanical model, based on continuum mechanics and on a volumetric tetrahedral Finite Element Method (FEM), is employed. This model can be suitably used for real-time applications through the SOFA simulator. Besides, an explicit physical modelling would enable a reliable prediction of internal forces undergone by the object.

In order to model elastic deformations, the infinitesimal strain theory and Hooke's law are taken into account, providing a linear relation between the displacement of the tetrahedral elements of the mesh and the internal forces exerted on their nodes. The co-rotational approach is used as a good compromise between the ability to model large deformations of the elements and computational efficiency.

Based on the FEM co-rotational model, fractures in the mesh are detected by decomposing the internal forces on the nodes into tensile and compressive forces to measure pure tensile forces acting on each node, through a so-called separation tensor. The fracture is propagated by simply removing attached elements intersected by the fracture plane. The model is illustrated in Fig. 3. 


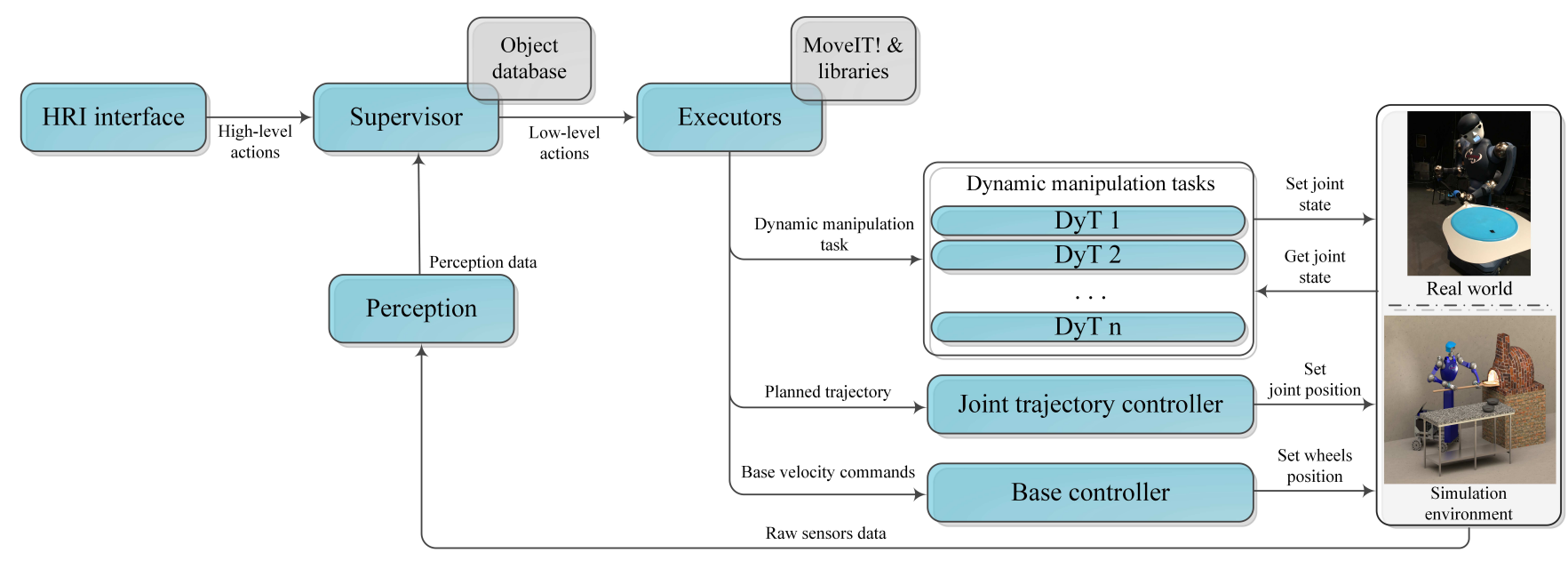

Fig. 2: RoDYMAN high-level control architecture.

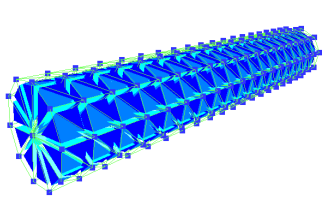

(a)

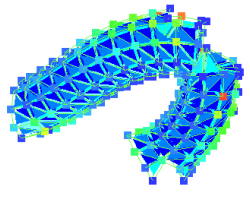

(b)
Fig. 3: Volumetric tetrahedral mesh (elements in blue colors) in (a), and modeling of fractures in (b).

The frame-by-frame tracking framework in Fig. 4 relies on a prior visual segmentation of the object in the image, based on a graph-cut based segmentation technique using color cues. The corresponding segmented point cloud is first registered through a classical Iterative Closest Point (ICP) method, and then by fitting the known mesh of the object on the point cloud. The basic idea is to derive external forces exerted by the point cloud on the mesh and to integrate them with the internal forces computed using the physical model into Lagrangian mechanical equations: $\boldsymbol{M} \ddot{\boldsymbol{x}}+\boldsymbol{C} \dot{\boldsymbol{x}}+\boldsymbol{K}^{\prime} \boldsymbol{x}+\boldsymbol{f}_{0}=\boldsymbol{f}_{\text {ext }}$, where $\boldsymbol{x} \in \mathbb{R}^{n}$ contains the positions of the $n$ vertices, $\boldsymbol{M} \in \mathbb{R}^{n \times n}$, $\boldsymbol{C} \in \mathbb{R}^{n \times n}$ and $\boldsymbol{K}^{\prime} \in \mathbb{R}^{n \times n}$ are the mass, damping and stiffness matrices, $\boldsymbol{f}_{\text {ext }} \in \mathbb{R}^{n}$ is the external forces vector, and $\boldsymbol{f}_{0} \in \mathbb{R}^{n}$ is an offset on the internal forces due to rotational effects. An Euler implicit integration scheme and a conjugate gradient method are used to solve the system with respect $\boldsymbol{x}$. The elastic forces $f_{\text {ext }}$ based on geometrical correspondences between the point cloud and the mesh [17].

To validate the method, some results have been obtained with various soft objects, deformations due to bending, stretching or compression actions, and fractures, and under challenging conditions, like occlusions or fast motions (see Fig. 5). Some preliminary experiments integrating the method into a robotic manipulation task of a silicon pizza dough, have also been carried out (see Fig.5c and Section IV-B).

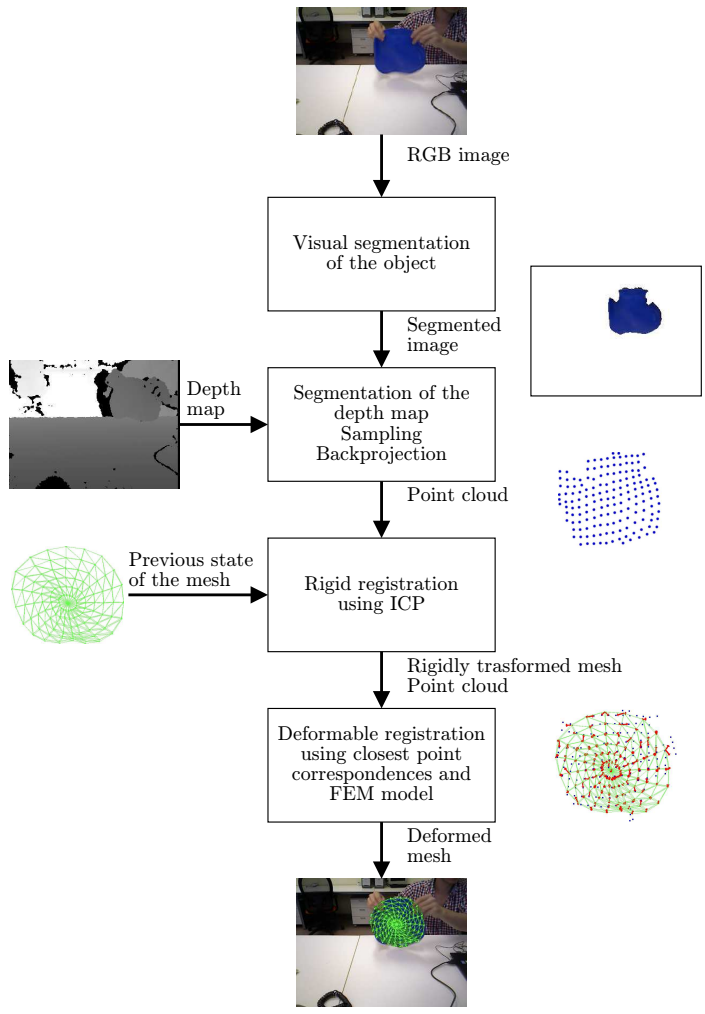

Fig. 4: Overview of the developed approach for deformable object tracking.

\section{NONPREHENSILE OBJECTS MOTION PLANNING AND CONTROL}

Four manipulation primitives have been considered so far within the RoDYMAN project. Namely, nonprehensile rolling, sliding, tossing and batting/juggling. Sliding and tossing take into account deformable objects, while rolling and batting/juggling only rigid ones. It is indeed difficult to find relevant applications involving deformable objects in pure rolling and juggling tasks. In the following, the controller and/or the motion planner designed for the aforementioned primitives are described. 


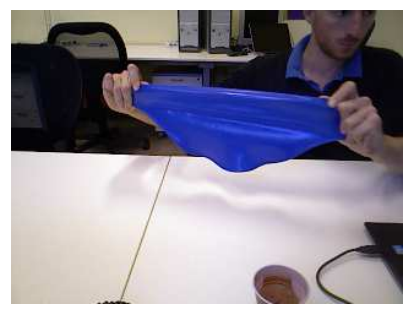

(a)

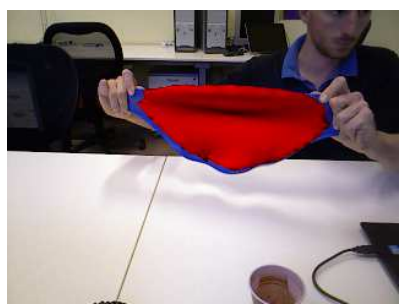

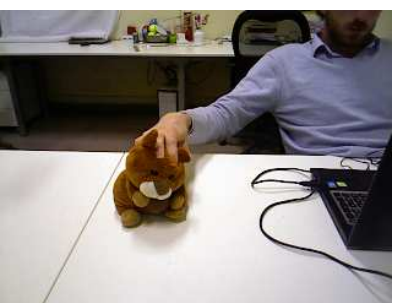

(b)

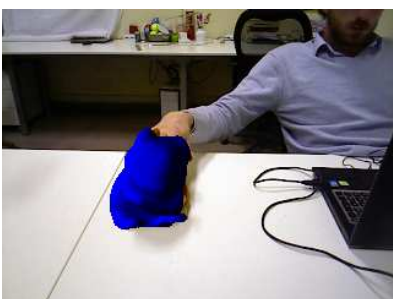

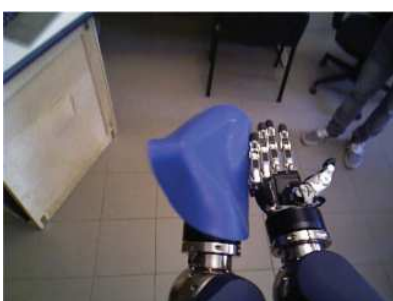

(c)

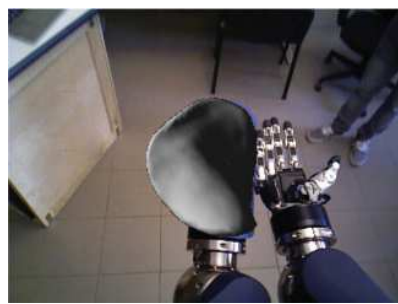

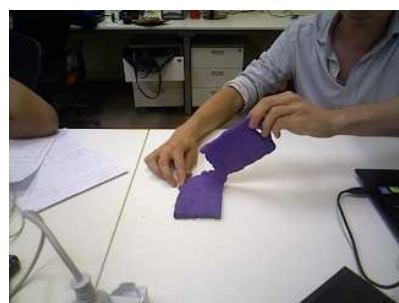

(d)

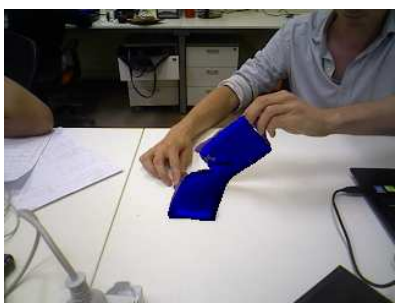

Fig. 5: Results of the tracking process with the input images (4 different objects in the first row), and the corresponding registered re-projected mesh (second row, in red for object (b), in blue for objects (b) and (d), in white for object (c)).

\section{A. Nonprehensile rolling}

An actuated manipulator of a given shape, referred to as hand, manipulates an object only through purely rotations, without grasping or caging it. Therefore, the object can only roll upon the shape of the hand. Case studies like the manipulation of a ball on a plate, the ball on a beam, and so on, are deeply examined in the literature. In this article, only planar rolling is described.

Since highly-geared harmonic drives are present within the RODYMAN mechatronic platform, it is suitable to suppose the acceleration of the hand $\ddot{\theta}_{h} \in \mathbb{R}$ as input $a_{h} \in \mathbb{R}$ for the system. The dynamic model for a nonprehensile planar rolling manipulation system, in which the hand can only rotate around its center of mass, is described by

$$
\begin{aligned}
& \ddot{\theta}_{h}=a_{h}, \\
& \ddot{s}_{h}=-b_{22}^{-1}\left(b_{12} a_{h}+c_{21} \dot{\theta}_{h}+c_{22} \dot{s}_{h}+g_{2}\right),
\end{aligned}
$$

where $s_{h} \in \mathbb{R}$ is the contact position of the object on the hand, whose shape is parametrized through arclength, $b_{12} \in \mathbb{R}$ and $b_{22} \in \mathbb{R}$ are entries of the inertia matrix $\boldsymbol{B} \in \mathbb{R}^{2 \times 2}$, while $c_{21} \in \mathbb{R}$ and $c_{22} \in \mathbb{R}$ are entries of the $(2 \times 2)$ Coriolis matrix of the mechanical system, and $g_{2} \in \mathbb{R}$ is the second element of the $(2 \times 1)$ gravity-force vector. Detailed expressions of each term are provided in [18], where it is also noticed that if the Coriolis terms are zero, a nonprehensile planar rolling manipulation system is differentially flat with the output $\frac{b_{12}}{b_{22}} \theta_{h}+s_{h}$. Among the class of systems for which the aforementioned assumption is true, it is worth recalling the ball-on-disk (BoD) system, which is mathematically equivalent to the disk-on-disk (DoD) system in the transversal plane [11].

The BoD consists of a ball rolling on a disk and arranged one on top of the other as shown in Fig 6. The disk is the actuated hand and the ball rolling on the hand is the object. The control problem for the $\mathrm{BoD}$ is to balance the object at the

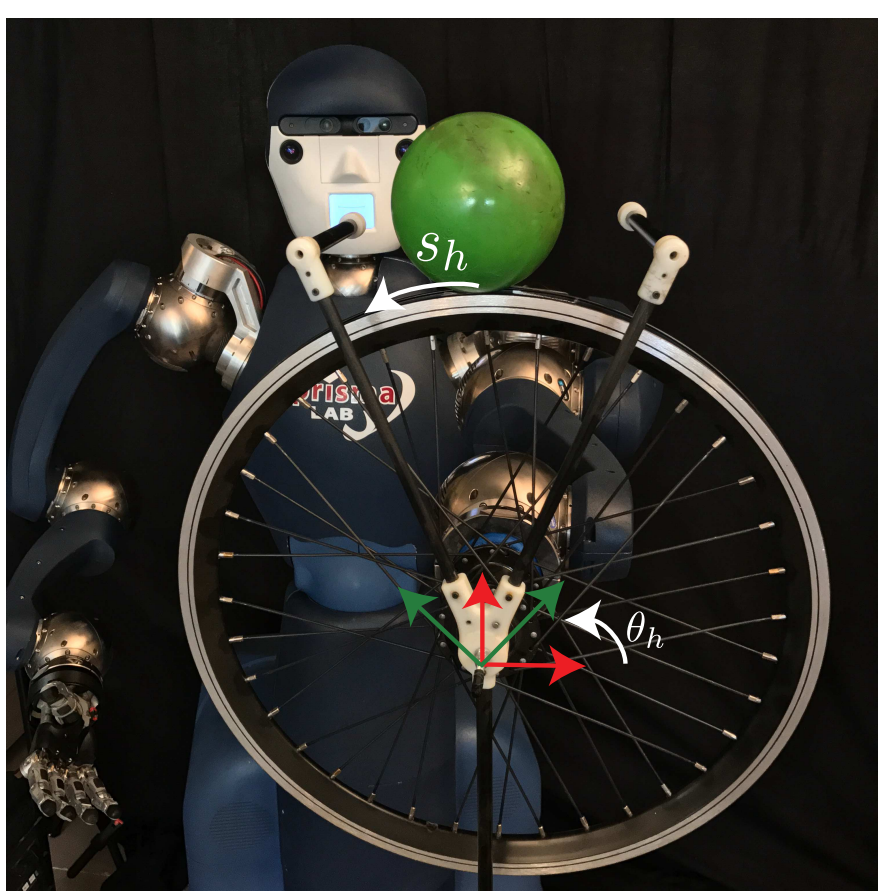

Fig. 6: The RoDyMAN platform actuating the BoD system. The disk is actuated by the movement of the RoDYMAN joints. The displayed structure, made by three connected bars, is employed to make possible to start the experiments with the ball in a position which is different from the desired equilibrium on the top of the disk. The world frame is depicted in red, the one attached to the rotating wheel is in green, $\theta_{h}$ represents the angle between the two, while $s_{h}$ measures the contact position of the ball on the wheel. 
upright position while driving the hand to a desired angular set-point. This problem is solved using passivity-based control (PBC) for port-Hamiltonian $(\mathrm{pH})$ systems. In its standard form, this approach applied to nonprehensile rolling aims at finding a control law for system (1a)-(1b) such that the closed-loop dynamics can be written as follows

$$
\left[\begin{array}{c}
\dot{\boldsymbol{q}} \\
\dot{\boldsymbol{p}}
\end{array}\right]=\left[\begin{array}{cc}
0 & \boldsymbol{B}^{-1} \boldsymbol{B}_{d} \\
-\boldsymbol{B}_{d} \boldsymbol{B}^{-1} & \boldsymbol{J}_{2}(\boldsymbol{q}, \boldsymbol{p})-\boldsymbol{R}_{d}(\boldsymbol{q}, \boldsymbol{p})
\end{array}\right] \nabla H_{d}(\boldsymbol{q}, \boldsymbol{p}),
$$

where $\boldsymbol{q} \in \mathbb{R}^{2}$ and $\boldsymbol{p} \in \mathbb{R}^{2}$ are the generalized coordinate and moment vectors, $H_{d}=\frac{1}{2} \boldsymbol{p}^{\top} \boldsymbol{B}_{d}^{-1}(\boldsymbol{q}) \boldsymbol{p}+V_{d}(\boldsymbol{q}) \in \mathbb{R}$ is the desired total energy of the closed-loop system, $\boldsymbol{B}_{d} \in \mathbb{R}^{2 \times 2}$ and $V_{d} \in \mathbb{R}$ are the desired mass matrix and potential energy, respectively, and $\boldsymbol{J}_{2} \in \mathbb{R}^{2 \times 2}$ and $\boldsymbol{R}_{d} \in \mathbb{R}^{2 \times 2}$ represent the gyroscopic forces and damping injection of the closed loop, respectively. The objective is to shape the desired energy of the closed-loop dynamics to get a minimum of the potential energy at the desired equilibrium. The asymptotically stability of the closed loop is ensured by using the desired energy as Lyapunov function and the detectability of the passive output. The full development of the control design is reported in [11] for the DoD example.

Within the RODYMAN project, control laws related to the nonprehensile rolling primitive have been developed also for the $3 \mathrm{D}$ case, like the stabilization of a ball on a flat plate and the control of a robotic hula-hoop [19]. As a milestone, it is possible to affirm that nonprehensile rolling can be successfully modelled through the $\mathrm{pH}$ formalism and consequently controlled with PBC approaches. This is relevant because it means that there exists a unified framework at least for such class of nonprehensile manipulation primitive.

\section{B. Friction-induced manipulation primitive}

As a case study, in order to uniformly cook a pizza, the dough must be rotated through a peel inside a wooden oven, in which the heat source is present only in a side of the structure. Similar actions are performed by chefs when some food must be brown or rotated in a pan.

From a dynamic point of view, friction plays a key role because of the sliding manipulation primitive between the tool and the part to be manipulated. In the literature, frictioninduced manipulation has been extensively studied to create virtual velocity fields on a vibrating plate actuated by a mechanical system equal or similar to a Stewart platform [10]. A similar concept has been suitably modified for the pizza case [20].

The RoDYMAN platform has successfully achieved a bimanual nonprehensile manipulation task through sliding by handling a peel to rotate the pizza placed on it. With reference to Fig. 1, the peel is chosen to be only translated (and rotated) along (and around) its longitudinal direction. A suitable combination of these two movements creates the desired motion of the object on the peel: an acceleration along the longitudinal direction moves the object back and forth on the peel once static friction is overcome; while, an angular acceleration around the same axis creates a non-uniform pressure distribution on the object. This, together with the linear acceleration, creates a rotation of the object. It is worth noticing that the object rotation is not decoupled from a linear displacement on the peel. Adaptations from [20] have been necessary to apply the concepts on the RODYMAN platform. Namely, two suitable smooth sinusoidal accelerations, with the same tunable frequency and different tunable amplitudes and phases, are planned for the linear and angular accelerations of the peel. The motion of the RoDYMAN joints are then retrieved by means of a standard closed-loop inverse kinematic algorithm. The above-described tracking of deformable objects has been employed to control the center of mass of the pizza towards the center of the peel through a simple PI-controller, while a complete rotation of the circular shape is requested.

Friction estimation is crucial within this task and several tests have been performed to suitably tune all the parameters of the control model to fit the real set-up. Current work aims at finding structural properties for the controller, like the design of orbital stabilization for the object on the peel (i.e., reach a desired rotational velocity).

\section{Tossing task}

The problem of tossing and catching a deformable object, like a pizza dough, is a procedure that is frequently dexterously performed by human pizza chefs. There are at least three reasons why tossing the dough during the preparation of the pizza is attractive: (i) the dough is stretched to a desired size, (ii) the dough naturally assumes a configuration that is thicker at the ends and thinner in the middle, and (iii) as the spinning dough freely falls, the outside of the dough dries, making it crunchy in the outside but light in the middle. The pizza chef is trained to perform a streamlined hand motion to toss and catch the dough, while a similar feat is a desired achievement for the RODYMAN robot.

The combined model of the dough grasped with robotic fingers through unilateral constraints, and the kinematics and dynamics of the robot manipulator has been derived in [4]. Upon that, a control law achieving the desired tossing motion can be designed. Furthermore, with a perfect knowledge of the motion of the dough, optimal trajectories can be generated in $S E(3)$ for the catching phase. The optimal trajectory generation is repeated as new sensor information is available. The trajectories are generated in such a way that the initial position, velocity, acceleration and final velocity and accelerations are matched, therefore, it is at least thrice continuously differentiable. An optimal trajectory, whose initial and final accelerations are prescribed, has to satisfy a sixthorder Boundary Value Problem (BVP). Such BVP is generated by using the necessary conditions for a path to minimize a convex combination of the jerk and acceleration functionals. While minimizing the jerk functional reduces the vibrations in the structure of the robotic manipulator, minimizing the acceleration functional reduces the total amount of energy expended during the catching motion. Only the case where the final position is left free and is part of the minimization problem is considered. More details can be found in [4].

Experimental validations are in progress. Nevertheless, preliminary results show that such kind of task requires high 
peak currents in the motors to toss the dough for more than $10 \mathrm{~cm}$. As anticipated above, the motors of RoDYMAN do not have such skills. As a matter of fact, analogies between tossing and walking gaits can be found within mathematical models. Similarly to robotic legs, hydraulic actuators seem to be more performing and the same might hold for tossing tasks. The stretching-the-dough task can be also performed in an alternative form which will be explored in the future.

\section{Batting/Juggling skills}

A very challenging primitive from the control view point is the one involving impacts. Inside batting, for instance, an object (a ball) is intercepted by the end-effector (a paddle) without grasping it, and it is thrown towards a precise goal. This motion primitive is typically used by athletes, such as baseball or table tennis players. Also jugglers use such primitive when their hands control the continuous motion of one or more objects through intermittent contacts. These dynamic motions require high velocity and precision. The design of planning and control methods to deal with them would strongly enhance capabilities of robot manipulators, extending the workspace size and enhancing dexterity.

The batting task dynamics is typically defined as hybrid, since it consists of the continuous aerodynamics of the manipulated ball (a differential equation), and the discontinuous reset of the velocity at impact time (two difference equations), given by

$$
\begin{aligned}
\ddot{\boldsymbol{p}}_{b} & =-\boldsymbol{g}-k_{d}\left\|\dot{\boldsymbol{p}}_{b}\right\| \dot{\boldsymbol{p}}_{b}+k_{l} \boldsymbol{S}\left(\boldsymbol{\omega}_{b}\right) \dot{\boldsymbol{p}}_{b} \\
\dot{\boldsymbol{p}}_{b}^{+} & =\boldsymbol{v}_{p}+\boldsymbol{\Gamma}_{a}\left(\dot{\boldsymbol{p}}_{b}^{-}-\boldsymbol{v}_{p}\right)+\boldsymbol{\Gamma}_{b} \boldsymbol{\omega}_{b}^{-} \\
\boldsymbol{\omega}_{b}^{+} & =\boldsymbol{\Gamma}_{c}\left(\dot{\boldsymbol{p}}_{b}^{-}-\boldsymbol{v}_{p}\right)+\boldsymbol{\Gamma}_{d} \boldsymbol{\omega}_{b}^{-}
\end{aligned}
$$

where $\boldsymbol{p}_{b} \in \mathbb{R}^{3}$ and $\boldsymbol{\omega}_{b} \in \mathbb{R}^{3}$ are the position and the spin of the ball, respectively; $\boldsymbol{v}_{p} \in \mathbb{R}^{3}$ is the paddle velocity; $\boldsymbol{\Gamma}_{j}\left(\boldsymbol{R}_{p}\right) \in \mathbb{R}^{3 \times 3}, j=\{a, b, c, d\}$, are transformation matrices dependent on the rebound parameters and on the orientation of the paddle $\boldsymbol{R}_{p} \in S O(3)$ at the impact time; $k_{d}\left(\dot{\boldsymbol{p}}_{b}, \boldsymbol{\omega}_{b}\right) \in \mathbb{R}$ and $k_{l}\left(\dot{\boldsymbol{p}}_{b}, \boldsymbol{\omega}_{b}\right) \in \mathbb{R}$ are, respectively, drag and lift parameters; $\boldsymbol{g} \in \mathbb{R}^{3}$ is gravity acceleration vector; $\|\cdot\|$ denotes the Euclidean norm; $\boldsymbol{S}(\cdot) \in \mathbb{R}^{3 \times 3}$ is the skewsymmetric matrix, while superscripts - and + represent the state before and after the impact, respectively. The matrices $\boldsymbol{\Gamma}_{j}$ can be detailed on the basis of the addressed rebound (ball impacting the table and/or a rubber paddle). Their expression may become complicated in non-trivial situations (like nonspherical objects): this leads to the use of some (string) assumptions and model reductions.

Five different phases have been considered to solve the batting problem by using the RODYMAN platform simulator. Firstly, a vision system is assumed to measure the trajectory of the ball. By assigning the impact time, the prediction of the impact position and pre-impact velocity of the ball are obtained numerically solving its aerodynamic model (3a). Then, the post-impact velocity of the ball, such that it goes towards a desired goal in a predefined time, is computed solving equation (3a) backward in time. The configuration of the paddle to generate such velocity of the ball results from the analytic solution of the discontinuous part of the ball-paddle model,

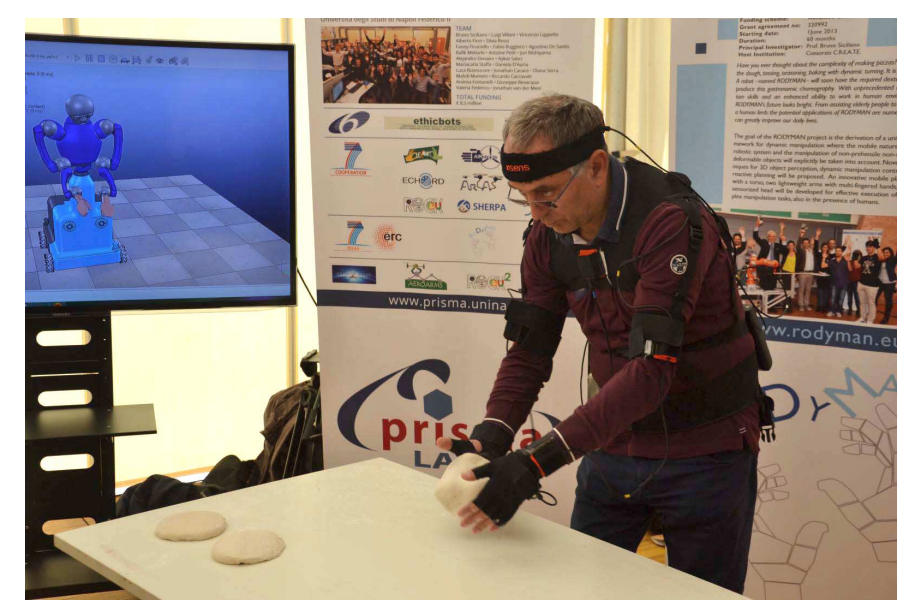

Fig. 7: The renowned pizza chef Enzo Coccia wearing the Xsens MVN suite, with the RODYMAN avatar in the background acquiring and repeating the movements of the chef.

given by the difference equations (3b) and (3c). Thereafter, the motion of the paddle to reach the desired configuration is a result of the minimization of its linear and angular acceleration with a coordinate-free approach, assuming that the path is generated on an arbitrary Riemannian manifold, similarly to the tossing primitive. Finally, the motion of the RODYMAN joints is derived from a classical second-order closed-loop kinematic inversion. More details can be found in [6].

A similar algorithm can be thought to accomplish different juggling patterns. While doing this, the lesson learned is that these techniques may also be applied to other dynamic tasks which share the same hybrid nature with impacting manipulations, such as walking or running tasks.

\section{FINAL DISCUSSION}

Despite several progresses have been addressed within the RODYMAN project so far, like real-time tracking of deformable objects employed in tossing and sliding tasks, several issues have to be tackled yet. The mechatronic platform should be revised to cope with issues given by the high-velocity of some nonprehensile manipulation primitives. In general, experiments involving nonprehensile actions are not easy to solve due to the uncertain dynamics mainly due to friction: parameters estimation and/or robust controllers are thus essential. Moreover, physics terms causing non-smooth behaviour are often neglected when deriving the mathematical model of a given nonprehensile task: this makes the nonprehensile system look like a prehensile one. This happens for instance within rolling, sliding and pushing nonprehensile manipulation primitives. The proof that the designed controller does not violate the given assumptions is usually performed a-posteriori. A method to directly control the contact forces should be indeed addressed and this might be a future research direction, leading to the design of non-smooth and hybrid controllers which are also a new frontier for the research community.

Another approach might instead be the observation of pizza chef activities to learn task simplification and synthesize human-inspired control strategies. For instance, an integrated 
robotic platform able to acquire and transfer human body motion to a robotic system is obtained by interfacing RODYMAN with a low-cost motion capture system (see Fig. 7). Once the teleoperation algorithm for real-time replication of human motion on RODYMAN is developed, a comprehensive taxonomy of dynamic prehensile and non-prehensile tasks, ordered for different levels of hand-arm and dual-arm coordination, can be built from scratch. To this aim, taking inspiration from the researches conducted on anthropomorphic hands [21] or a single hand-arm system [22], a study on postural synergies for dual-arm robotic manipulation can be conducted to develop a framework where to simplify learning strategies from human imitation. Such approach will also take advantage of dimensionality reduction strategy to successfully apply supervised reinforcement learning algorithms using synergistic motion. Today, from these observations, it has been learned that the motion planner is crucial for nonprehensile tasks since the repetitive actions seem well imprinted in the pizza chef's mind, while the corrections made by the hands are very small despite the difference between various doughs. Therefore, it is reasonable to state that a good motion planner is the essential instrument within nonprehensile manipulation.

One further question that may arise is: why has the pizzamaking procedure been taken as example? Is there the need of having a robot making pizza? In truth, the pizza-making process is only a media expedient with scientific purposes. It is indeed clear that if a robot is able to manipulate a pizza dough, it might be able to perform similar difficult manipulation tasks. For instance, in 1997 the RoboCup started certainly not to replace the real soccer players, but rather to advance the state of the art while facing both gaming and difficult problems for robots. With the same aim, RoDYMAN is trying to mimic the artistic ability of a pizza chef. While facing this big challenge, many sub-problems have to be addressed in parallel which could have an impact in other domains. The perception of elastic objects is currently being applied in the medical context to shape variations of muscles and organs. The manipulation performed while tossing the deformable dough is currently under investigation to improve the automation of gluing the shoes' lower surfaces. The batting process has similar dynamics to the walking gaits, and as such it could be used to improve autonomy of humanoids, or employed for actuated prostheses.

\section{ACKNOWLEDGMENT}

The research leading to these results has been supported by the RoDyMan project, which has received funding from the European Research Council FP7 Ideas under Advanced Grant agreement number 320992. The authors are solely responsible for the content of this manuscript.

\section{REFERENCES}

[1] D. Prattichizzo and J. Trinkle, "Grasping," in Springer Handbook of Robotics, B. Siciliano and O. Khatib, Eds. Springer International Publishing, 2016, pp. 955-988.

[2] R. M. Murray, Z. Li, and S. S. Sastry, A mathematical introduction to robotic manipulation. CRC press, 1994.
[3] K. Lynch and T. D. Murphey, "Control of nonprehensile manipulation," in Control Problems in Robotics, ser. Springer Tracts in Advanced Robotics, A. Bicchi, D. Prattichizzo, and H. Christensen, Eds. Springer Berlin Heidelberg, 2003, vol. 4, pp. 39-57.

[4] A. Satici, F. Ruggiero, V. Lippiello, and B. Siciliano, "A coordinatefree framework for robotic pizza tossing and catching," in 2016 IEEE International Conference on Robotics and Automation, Stockholm, S, 2016, pp. 3932-3939.

[5] G. Bätz, A. Yaqub, H. Wu, K. Kuhnlenz, D. Wollherr, and M. Buss, "Dynamic manipulation: Nonprehensile ball catching," in 18th Mediterranean Conference on Control and Automation, Marrakech, MA, 2010, pp. $365-370$.

[6] D. Serra, A. Satici, F. Ruggiero, V. Lippiello, and B. Siciliano, "An optimal trajectory planner for a robotic batting task: the table tennis example," in 13th International Conference on Informatics in Control, Automation and Robotics, Lisbon, P, 2016, pp. 90-101.

[7] P. Reist and R. D'Andrea, "Design and analysis of a blind juggling robot," IEEE Transactions on Robotics, vol. 28, no. 6, pp. 1228-1243, 2012.

[8] G. Bätz, U. Mettin, A. Schimdts, M. Scheint, D. Wollherr, and A. Shiriaev, "Ball dribbling with an underactuated continuous-time control phase: Theory \& experiments," in 2010 IEEE/RSJ International Conference on Intelligent Robots and Systems, Taipei, Taiwan, 2010, pp. 2890-2895.

[9] K. Lynch and M. Mason, "Stable pushing: Mechanics, controllability, and planning," The International Journal of Robotics Research, vol. 15, no. 6, pp. 533-556, 1996.

[10] T. Vose, P. Umbanhowar, and K. Lynch, "Friction-induced velocity fields for point parts sliding on a rigid oscillated plate," The International Journal of Robotics Research, vol. 28, no. 8, pp. 1020-1039, 2009.

[11] A. Donaire, F. Ruggiero, L. Buonocore, V. Lippiello, and B. Siciliano, "Passivity-based control for a rolling-balancing system: The nonprehensile disk-on-disk," IEEE Transactions on Control Systems Technology, 2016, in press.

[12] J. Woodruff and K. Lynch, "Planning and control for dynamic, nonprehensile, and hybrid manipulation tasks," in 2017 IEEE International Conference on Robotics and Automation, Singapore, 2017, pp. 40664073.

[13] C. D. Sousa and R. Cortesão, "Physically feasibility of robot base inertial parameters identification: A linear matrix inequality approach," International Journal of Robotics Research, vol. 33, no. 6, pp. 931-944, 2014.

[14] A. De Luca, A. Albu-Schaffer, S. Haddadin, and G. Hirzinger, "Collision detection and safe reaction with the DLR-III lightweight manipulator arm," in 2006 IEEE/RSJ International Conference on Intelligent Robots and Systems, Beijing, C, 2006, pp. 1623-1630.

[15] J. Cacace, A. Finzi, V. Lippiello, G. Loianno, and D. Sanzone, "Aerial service vehicles for industrial inspection: Task decomposition and plan execution," Applied Intelligence, vol. 42, no. 1, pp. 49-62, 2015.

[16] S. Chitta, I. Sucan, and S. Cousings, "MoveIt! [ROS topics]," IEEE Robotics \& Automation Magazine, vol. 19, no. 1, pp. 18-19, 2014.

[17] A. Petit, V. Lippiello, G. A. Fontanelli, and B. Siciliano, "Tracking elastic deformable objects with an RGB-D sensor for a pizza chef robot," Robotics and Autonomous Systems, vol. 88, pp. 187-201, 2017.

[18] V. Lippiello, F. Ruggiero, and B. Siciliano, "The effects of shapes in input-state linearization for stabilization of nonprehensile planar rolling dynamic manipulation," Robotics and Automation Letters, vol. 1, no. 1, pp. 492-499, 2016.

[19] A. Gutirrez-Giles, F. Ruggiero, V. Lippiello, and B. Siciliano, "Modeling and control of a robotic hula-hoop system without velocity measurements," in 20th World Congress of the International Federation of Automatic Control, Toulouse, F, 2017.

[20] M. Higashimori, K. Utsumi, Y. Omoto, and M. Kaneko, "Dynamic manipulation inspired by the handling of a pizza peel," IEEE Transactions on Robotics, vol. 25, no. 4, pp. 829-838, 2009.

[21] F. Ficuciello, G. Palli, C. Melchiorri, and B. Siciliano, "Postural synergies of the UB Hand IV for human-like grasping," Robotics and Autonomous Systems, vol. 62, no. 4, pp. 515-527, 2014.

[22] F. Ficuciello, D. Zaccara, and B. Siciliano, "Synergy-based policy improvement with path integrals for anthropomorphic hands," in 2016 IEEE/RSJ International Conference on Intelligent Robots and Systems, Daejeon, K, 2016, pp. 1940-1945. 